\title{
The POD Model Order Reduction Method of Accelerating Reservoir Numerical Simulation
}

\author{
CAO Jing, ZHAO Hui, YU Gaoming, XIE Yunhong \\ (College of Petroleum Engineering, Yangtze University, Wuhan 430100, China)
}

\begin{abstract}
Because the reservoir simulation models arising from real field may consist of hundreds of thousands or millions of grid blocks, traditional reservoir simulators can be quite time-consuming. This also led to computational cost of production optimization and history matching process of closed-loop reservoir management be greatly increased. Improving reservoir simulation speed is the urgent problem to be solved. In this paper, the proper orthogonal decomposition (POD) model order reduction method is applied in reservoir numerical simulation. First of all, in the training process, full order reservoir simulator is run to get snapshots and the POD base matrix is constructed. Then the base matrix is reused to predict under different production schedule. Numerical experiment shows that this method can greatly reduce the dimensions of reservoir model and improve the operation speed of simulator by nearly three times under the conditions with sufficient precision to ensure, and verify the validity of proposed method.
\end{abstract}

Keywords: reservoir numerical simulation; fully implicit; model order reduction; proper orthogonal decomposition (POD); control system

Reservoir numerical simulation is one of the important means of reservoir development. It can make engineers better understand the reservoir physical properties and fluid flow law, in order to make the correct evaluation and determine the reasonable development plan and measures to improve the recovery rate. In the traditional reservoir simulator, the partial differential equations, which are composed of the equations of motion, the state equation and the continuity equation, are discretized and transformed into a set of nonlinear algebraic equations, and then solved by iterative method. However, for the actual oil reservoir model may include hundreds of thousands or millions of grid, number of equations required is very large, the traditional reservoir simulator is very time-consuming. In particular, when the reservoir simulator is used in the closed-loop reservoir management [1-5], production optimization and the history matching process require repeated operation simulator. This leads to a significant increase in computational cost, which is also an important bottleneck that can not be applied in a large scale. Therefore, in order to ensure the accuracy of the numerical solution, how to accelerate the simulation speed of the reservoir is an urgent problem to be solved.

The model reduction technique was first used in the field of automatic control and circuit system. Its task is to reduce the dimension of the state space vector and keep the input and output characteristics of the system at the same time. The proper orthogonal decomposition method (POD) is the most widely used in nonlinear system model reduction method. The computational cost of this method is low, and it has some stability, maintains some basic properties of the original system. The POD method has been successfully applied to computational fluid mechanics [6], structural mechanics [7], and meteorology [8], digital signal processing [9], and other fields. In this paper, the POD model reduction method is applied to reservoir simulator, which can greatly reduce the dimension of reservoir model, so as to reduce the calculation time and improve the operation speed.

\section{Control Equation of Reservoir Model}

In this paper, the mathematical model of reservoir model is transformed into the state space equation by means of space discrete in order to explain the reduction process of POD method. Two dimensional oil-water two phase reservoir model is used. It is assumed that oil and water do not exchange material, the process is isothermal, the fluid is compressible, and the mass conservation equation and Darcy's law can be used to obtain [10]:

$$
-\nabla \bullet\left[\frac{k_{r i} \rho_{i}}{\mu_{i}} \mathbf{K}\left(\nabla p_{i}-\rho_{i} g \nabla d\right)\right]+\frac{\partial\left(\phi S_{i} \rho_{i}\right)}{\partial t}-\rho_{i} q_{i}{ }^{\prime \prime}=0
$$

Where $\mathbf{K}$ is permeability tensor; $\mu$ is fluid viscosity; $k_{r}$ is relative permeability; $p$ is pressure; $g$ is gravity acceleration; $d$ is depth; fluid density; $\phi$ is porosity; $S$ is fluid saturation; $t$ is time; $q^{\prime \prime \prime}$ is a source term expressed as flow rate per unit volume; superscript $i \in\{o, w\}$ is respectively oil phase and water phase. In the 
equation (1), there are four unknown quantities, $p_{w}$ and $S_{o}$ are eliminated by using the auxiliary equation (2) and (3), so that only the state variables $p_{o}, S_{w}$ are included in the equation,

$$
\begin{aligned}
& S_{o}+S_{w}=1 \\
& p_{o}-p_{w}=p_{c}\left(S_{w}\right)
\end{aligned}
$$

Where $p_{c}\left(S_{w}\right)$ is oil-water two-phase capillary pressure.

We consider the relatively simple cases and ignore gravity and capillary force. Format to discrete in space by using five point block centered finite difference, we may have the nonlinear first-order differential equation (4), see the specific derivation of literature [11]:

$$
\underbrace{\left[\begin{array}{ll}
\mathbf{V}_{w p} & \mathbf{V}_{w s} \\
\mathbf{V}_{o p} & \mathbf{V}_{o s}
\end{array}\right]}_{\mathbf{V}}\left[\begin{array}{c}
\dot{\mathbf{p}} \\
\dot{\mathbf{s}}
\end{array}\right]+\underbrace{\left[\begin{array}{ll}
\mathbf{T}_{w} & \mathbf{0} \\
\mathbf{T}_{o} & \mathbf{0}
\end{array}\right]}_{\mathbf{T}}\left[\begin{array}{l}
\mathbf{p} \\
\mathbf{s}
\end{array}\right]=\underbrace{\left[\begin{array}{c}
\mathbf{F}_{w}(\mathbf{s}) \\
\mathbf{F}_{o}(\mathbf{s})
\end{array}\right]}_{\mathbf{F}} \mathbf{q}_{\text {well }, t}
$$

Where: vector $\mathbf{p}$ and $\mathbf{S}$ is grid center oil pressure $p_{o}$ and water saturation $S_{w}$ respectively; $\dot{\mathbf{p}}$ and $\dot{\mathbf{S}}$ is the time $t$ derivative of vector $\mathbf{p}$ and $\mathbf{S}$ respectively; $\mathbf{V}$ is the cumulative matrix; $\mathbf{T}$ is transmission matrix; $\mathbf{F}$ is divided flow matrix; Vector $\mathbf{q}_{\text {well }, t}$ is the total flow of oil-water well.

Define the state vector $\mathbf{X}$, input vector $\mathbf{u}$ and output vector $\mathbf{y}$

$$
\mathbf{x} \square\left[\begin{array}{l}
\mathbf{p} \\
\mathbf{s}
\end{array}\right] \quad \mathbf{u} \square\left[\begin{array}{l}
\breve{\mathbf{q}}_{\text {well,t }} \\
\breve{\mathbf{p}}_{\text {well }}
\end{array}\right] \quad \mathbf{y} \square\left[\begin{array}{l}
\overline{\mathbf{p}}_{\text {well }} \\
\overline{\mathbf{q}}_{\text {well }, w} \\
\overline{\mathbf{q}}_{\text {well }, \mathrm{o}}
\end{array}\right]
$$

Where vector $\breve{\mathbf{q}}_{\text {well }, t}$ and $\breve{\mathbf{p}}_{\text {well }}$ represent the well of the constant flow and the bottom hole pressure respectively; The vector $\overline{\mathbf{p}}_{\text {well }}$ indicates the output bottom hole flow pressure of the constant flow well; Vector $\overline{\mathbf{q}}_{\text {well }, \mathrm{o}}$ and $\overline{\mathbf{q}}_{\text {well,w }}$ indicate the output oil and water flow of the constant bottom hole pressure respectively.

The equation (4) can be written as the form of state space equation [11]:

$$
\begin{aligned}
& \dot{\mathbf{x}}=\mathbf{f}(\mathbf{x}, \mathbf{u})=\mathbf{A}_{\mathbf{c}}(\mathbf{x}) \mathbf{x}+\mathbf{B}_{\mathbf{c}}(\mathbf{x}) \mathbf{u} \\
& \mathbf{y}=\mathbf{h}(\mathbf{x}, \mathbf{u})=\mathbf{C}(\mathbf{x}) \mathbf{x}+\mathbf{D}(\mathbf{x}) \mathbf{u}
\end{aligned}
$$

In the control system, $\mathbf{A}_{\mathbf{c}}$ is called the system matrix, $\mathbf{B}_{\mathbf{c}}$ is called the input matrix, $\mathbf{C}$ is called the output matrix, $\mathbf{D}$ is called the direct transfer matrix. Because the elements of the matrix $\mathbf{V}, \mathbf{T}, \mathbf{F}, \mathbf{J}$ are function of the state variables, the system is a nonlinear system. we have

The control equation (8) is solved by the full implicit method, $\Delta \mathbf{x} / \Delta t$ approximating derivation $d \mathbf{x} / \mathrm{d} t$,

$$
\frac{\Delta \mathbf{x}}{\Delta t} \approx \mathbf{f}(\mathbf{u}(\mathrm{t}), \mathbf{x}(\mathrm{t}))
$$

Sets $\mathbf{x}_{k}$ representing the value of the state variable at $t_{k}, \mathbf{x}_{k-1}$ representing the value of the state variable at $t_{k-1}$, we have

Ordering $\mathbf{f}_{k}=\mathbf{x}_{k-1}+\mathbf{f}_{c, k} \Delta t$, there are

$$
\mathbf{x}_{k}=\mathbf{x}_{k-1}+\Delta t \mathbf{f}_{c}\left(\mathbf{u}_{k}, \mathbf{x}_{k}\right)
$$

$$
\mathbf{x}_{k}=\mathbf{f}_{k}\left(\mathbf{u}_{k}, \mathbf{x}_{k}, \mathbf{x}_{k-1}\right)
$$

Since both ends of the equation (12) contains $\mathbf{x}_{k}$, it is a nonlinear equation set, which is usually solved by Newton iteration method. However, when the dimension of the variables is very high, the solving process is very time-consuming, and the computational cost is large. For this purpose, the POD model reduction method is applied to the reservoir simulation, the dimension of $\mathbf{x}_{k}$ is reduced, and the operation speed is improved. 


\section{POD Model Reduction Methods}

POD method is also called empirical orthogonal function method and Karhunen-Loève decomposition method. In this method, a set of optimal orthogonal basis is constructed by using the data obtained from the given theory or experiment to reduce the order. The basic principle of the method and its application in reservoir numerical simulation are given below. The POD method is introduced in detail in the reference $[12,13]$.

\subsection{The basic principle of POD method [14]}

Assuming that $\varphi_{1}, \varphi_{2}, \cdots, \varphi_{\mathrm{n}}$ is the standard orthogonal basis vector of the data $\operatorname{set}\left\{\mathbf{x}_{1}, \mathbf{x}_{2}, \cdots, \mathbf{x}_{\mathbf{m}}\right\}$, the sample $\mathbf{x}_{\mathbf{i}} \in \mathbf{R}^{\mathbf{n}}$ can be linearly expressed in the following form

$$
\mathbf{x}_{\mathbf{i}}=c_{1 i} \boldsymbol{\varphi}_{\mathbf{1}}+c_{2 i} \boldsymbol{\varphi}_{\mathbf{2}}+\cdots+c_{n i} \boldsymbol{\varphi}_{\mathbf{n}}, \quad i=1,2, \cdots, m
$$

where $c_{j i}=\boldsymbol{\varphi}_{\mathbf{j}}{ }^{\mathrm{T}} \mathbf{x}_{\mathbf{i}}$. If the first $k$ base vector is chosen to approximate the sample vector $\mathbf{x}_{\mathbf{i}}$, we have

$$
\mathbf{x}_{\mathbf{i}(\mathbf{k})}=c_{1 i} \boldsymbol{\varphi}_{\mathbf{1}}+c_{2 i} \boldsymbol{\varphi}_{\mathbf{2}}+\cdots+c_{k i} \boldsymbol{\varphi}_{k}
$$

Ordering error function $\varepsilon^{2}=\sum_{i=1}^{m}\left\|\mathbf{x}_{\mathbf{i}}-\mathbf{x}_{\mathbf{i}(\mathbf{k})}\right\|_{2}^{2}$, there are

$$
\varepsilon^{2}=\sum_{i=1}^{m}\left\|\sum_{j=k+1}^{n} c_{j i} \boldsymbol{\varphi}_{\mathbf{j}}\right\|_{2}^{2}=\sum_{i=1}^{m} \sum_{j=k+1}^{n} c_{j i}^{2}=\sum_{i=1}^{m} \sum_{j=k+1}^{n} \boldsymbol{\varphi}_{\mathbf{j}}^{\mathbf{T}} \mathbf{x}_{\mathbf{i}} \mathbf{x}_{\mathbf{i}}^{\mathbf{T}} \boldsymbol{\varphi}_{\mathbf{j}}=\sum_{j=k+1}^{n} \boldsymbol{\varphi}_{\mathbf{j}}^{\mathbf{T}} \mathbf{X} \mathbf{X}^{\mathbf{T}} \boldsymbol{\varphi}_{\mathbf{j}} 。
$$

For the data set $\left\{\mathbf{x}_{1}, \mathbf{x}_{2}, \cdots, \mathbf{x}_{\mathbf{m}}\right\}$, POD goal is to find a set of optimal base vector $\left\{\varphi_{1}, \varphi_{2}, \cdots, \varphi_{n}\right\}$, in order to minimize the errors $\varepsilon^{2}$. The Lagrange factor $u_{i j}(i, j=k+1, k+2, \cdots, n)$ is introduced, and the Lagrange function is constructed

$$
L=\sum_{j=k+1}^{n} \boldsymbol{\varphi}_{\mathbf{j}}^{\mathbf{T}} \mathbf{X} \mathbf{X}^{\mathbf{T}} \boldsymbol{\varphi}_{\mathbf{j}}-\sum_{i=k+1}^{m} \sum_{j=k+1}^{n} u_{i j}\left(\boldsymbol{\varphi}_{\mathbf{i}}^{\mathbf{T}} \boldsymbol{\varphi}_{\mathbf{j}}-\delta_{i j}\right) 。
$$

$\varphi_{\mathrm{j}}$ 's partial derivative of the two ends of equation (16), there are

$$
\frac{\partial L}{\partial \boldsymbol{\varphi}_{\mathbf{j}}}=2\left(\mathbf{X} \mathbf{X}^{\mathbf{T}} \boldsymbol{\varphi}_{\mathbf{j}}-\sum_{i=k+1}^{m} u_{i j} \boldsymbol{\varphi}_{i}\right)=2 \mathbf{X} \mathbf{X}^{\mathbf{T}} \boldsymbol{\varphi}_{\mathbf{j}}-2 \boldsymbol{\Phi}_{\mathbf{n}-\mathbf{k}} \mathbf{u}_{\mathbf{j}},
$$

Where $\boldsymbol{\Phi}_{\mathbf{n}-\mathbf{k}}=\left[\boldsymbol{\varphi}_{k+1}, \boldsymbol{\varphi}_{k+2}, \cdots, \boldsymbol{\varphi}_{\mathbf{n}}\right], \quad \mathbf{u}_{\mathbf{j}}=\left[u_{k+1, j} u_{k+2, j} \cdots u_{\mathrm{n}, j}\right]^{T}$. Writing equation (17) in a more compact matrix form

$$
\frac{\partial L}{\partial \Phi_{n-k}}=2 \mathbf{X} \mathbf{X}^{\mathrm{T}} \boldsymbol{\Phi}_{\mathrm{n}-\mathrm{k}}-2 \boldsymbol{\Phi}_{\mathrm{n}-\mathrm{k}} \mathbf{U}_{\mathrm{n}-\mathrm{k}}
$$

Where $\mathbf{U}_{\mathbf{n}-\mathbf{k}}=\left[\mathbf{u}_{\mathbf{k}+1} \mathbf{u}_{\mathbf{k}+2} \cdots \mathbf{u}_{\mathbf{n}}\right]$. For the sake of the optimal solution, let the left end equation (18) is 0 , there are

$$
\mathbf{X} \mathbf{X}^{\mathrm{T}} \Phi_{\mathrm{n}-\mathrm{k}}=\Phi_{\mathrm{n}-\mathrm{k}} \mathbf{U}_{\mathrm{n}-\mathrm{k}}
$$

The both end of equation (19) premultiplication $\boldsymbol{\Phi}_{\mathbf{n}-\mathbf{k}}^{\mathrm{T}}$, getting $\mathbf{U}_{\mathbf{n}-\mathbf{k}}=\boldsymbol{\Phi}_{\mathbf{n}-\mathbf{k}}^{\mathbf{T}} \mathbf{X} \mathbf{X}^{\mathbf{T}} \boldsymbol{\Phi}_{\mathbf{n}-\mathbf{k}}$. Matrix $\mathbf{U}_{\mathbf{n}-\mathbf{k}}$ is a positive semidefinite matrix, so there is an orthogonal matrix $\mathbf{P}$ which makes the following equation established

$$
\mathbf{P}^{\mathrm{T}} \boldsymbol{\Phi}_{\mathrm{n}-\mathrm{k}}^{\mathrm{T}} \mathbf{X} \mathbf{X}^{\mathrm{T}} \boldsymbol{\Phi}_{\mathrm{n}-\mathrm{k}} \mathbf{P}=\mathbf{P}^{\mathrm{T}} \mathbf{U}_{\mathrm{n}-\mathrm{k}} \mathbf{P}=\Lambda
$$

Where $\Lambda$ is diagonal matrix. On the both ends of equation (19) right multiplication matrix $\mathbf{P}$, and the combination of equation (20), there is

$$
\mathbf{X X} \boldsymbol{\Phi}_{\mathrm{n}-\mathrm{k}} \mathbf{P}=\Phi_{\mathrm{n}-\mathrm{k}} \mathbf{P} \mathbf{P}^{\mathrm{T}} \mathbf{U}_{\mathrm{n}-\mathrm{k}} \mathbf{P}=\Phi_{\mathrm{n}-\mathrm{k}} \mathbf{P} \Lambda
$$

According to the formula (21), the diagonal elements of the matrix $\Lambda$ are composed of the eigenvalues $\lambda_{i}$ of the matrix $\mathbf{X X} \mathbf{X}^{\mathbf{T}}$, and the matrix $\Phi_{\mathbf{n}-\mathbf{k}} \mathbf{P}$ is composed of the eigenvectors corresponding to $\lambda_{i}$ of the matrix $\mathbf{X} \mathbf{X}^{\mathbf{T}}$. In fact, the diagonal elements of matrix $\Lambda$ are the square of the singular value $\sigma_{i}$ of the matrix $\mathbf{X}^{\mathbf{T}}$. Considering 
that the orthogonal matrix is guaranteed under the Frobenius norm $\|\bullet\|_{F}$, we get

$$
\varepsilon^{2}=\left\|\mathbf{X}^{\mathbf{T}} \boldsymbol{\Phi}_{\mathbf{n}-\mathbf{k}}\right\|_{F}^{2}=\left\|\mathbf{X}^{\mathbf{T}} \boldsymbol{\Phi}_{\mathbf{n}-\mathbf{k}} \mathbf{P}\right\|_{F}^{2}=\operatorname{trace}\left\{\left(\mathbf{X}^{\mathrm{T}} \boldsymbol{\Phi}_{\mathbf{n}-\mathbf{k}} \mathbf{P}\right)^{\mathbf{T}} \mathbf{X}^{\mathbf{T}} \boldsymbol{\Phi}_{\mathbf{n}-\mathbf{k}} \mathbf{P}\right\}=\operatorname{trace}\{\boldsymbol{\Lambda}\} 。
$$

In order to obtain the minimum error, the diagonal elements of the matrix $\Lambda$ need to select the last $n-k$ singular value of the matrix $\mathbf{X}^{\mathbf{T}}$. In this way, there will be $\min \varepsilon^{2}=\sum_{j=k+1}^{n} \sigma_{j}^{2}$.

The discussion concluded from above that, when choosing the right singular value vector $\left\{\mathbf{v}_{\mathbf{i}}\right\}_{i=1}^{k}$ of matrix $\mathbf{X}^{\mathbf{T}}$ as $k$ dimensional POD base vector $\left\{\boldsymbol{\varphi}_{\mathbf{i}}\right\}_{i=1}^{k}$, which not only satisfies the optimality conditions, and has obtained the minimum error. The minimum error is The minimum error is the square sum of the last $n-k$ singular values of the matrix.

Usually in the input / output system, the larger eigenvalues correspond to the main features of the system. We choose the $k$ POD basis vector $\left\{\boldsymbol{\varphi}_{\mathbf{i}}\right\}_{i=1}^{k}$ to represent the original vector more features. Setting $I(k)=\sum_{i=1}^{k} \lambda_{i} / \sum_{i=1}^{n} \lambda_{i}, \lambda_{i}(i=1,2, \cdots, n)$ is descending order eigenvalue of correlation matrix. If $I(k) \geq d \%, k$ POD basis vectors $\left\{\boldsymbol{\varphi}_{\mathbf{i}}\right\}_{i=1}^{k}$ maintain $d \%$ characteristic information of the original data $\operatorname{set}\left\{\mathbf{x}_{\mathbf{i}}\right\}_{i=1}^{k}$, which $k$ satisfies the

$$
k=\min \{i \mid I(k) \geq d \%, i=1,2, \cdots, n\} 。
$$

\subsection{Application of POD method in reservoir simulation}

In order to construct a POD basis vector, the first we need to run a full order reservoir simulator (also known as the training process), and to preserve the state vector $\mathbf{X}$ of each time step (also called the snapshot, including the oil pressure $p_{o}$ and the water saturation $S_{w}$ of all grids). Because of pressure and saturation with different physical properties, we use matrix $\mathbf{X}_{p}, \mathbf{X}_{S}$ to preserve $p_{o}, S_{w}$ respectively (hereinafter abbreviated as $p$ and $S$ ):

$$
\mathbf{X}_{p}=\left[\begin{array}{llll}
\mathbf{x}_{p}{ }^{1} & \mathbf{x}_{p}{ }^{2} \cdots & \mathbf{x}_{p}{ }^{m}
\end{array}\right], \quad \mathbf{X}_{S}=\left[\begin{array}{llll}
\mathbf{x}_{S}{ }^{1} \mathbf{x}_{S}{ }^{2} \cdots & \mathbf{x}_{S}{ }^{m}
\end{array}\right]
$$

Assuming that the number of grids in the reservoir model is $N$, then each vector $\mathbf{x}_{p}{ }^{i}, \mathbf{x}_{S}{ }^{i}$ (Superscript $i$ denotes the number of snapshots)in the matrix $\mathbf{X}_{p}$ and $\mathbf{X}_{S}$ is $N$ dimension, however, the dimension of the state vector $\mathbf{X}$ of the system is: $n=2 N$. The snapshot needs to be calculated mean value after the snapshot is obtained:

$$
\overline{\mathbf{x}}_{p}=\frac{1}{m} \sum_{i=1}^{m} \mathbf{x}_{p}{ }^{i}, \quad \overline{\mathbf{x}}_{S}=\frac{1}{m} \sum_{i=1}^{m} \mathbf{x}_{S}{ }^{i}
$$

And each snapshot in the data matrix $\mathbf{X}_{p}$ and $\mathbf{X}_{S}$ is subtracted from the mean:

$$
\begin{aligned}
\hat{\mathbf{X}}_{p} & =\left[\mathbf{x}_{p}{ }^{1}-\overline{\mathbf{x}}_{p}, \mathbf{x}_{p}{ }^{2}-\overline{\mathbf{x}}_{p}, \cdots, \mathbf{x}_{p}{ }^{m}-\overline{\mathbf{x}}_{p}\right] \\
\hat{\mathbf{X}}_{S} & =\left[\mathbf{x}_{S}{ }^{1}-\overline{\mathbf{x}}_{S}, \mathbf{x}_{S}{ }^{2}-\overline{\mathbf{x}}_{S}, \cdots, \mathbf{x}_{S}{ }^{m}-\overline{\mathbf{x}}_{S}\right]
\end{aligned}
$$

Implementing the above POD reduction process for the matrix $\hat{\mathbf{X}}_{p}$, the basis vector matrix $\boldsymbol{\Phi}_{l p}$ and $\boldsymbol{\Phi}_{l s}$ are obtained, and we combine the two matrices to obtain basis matrix $\boldsymbol{\Phi}_{l}$, which includes $l$ columns, and $l=l_{p}+l_{s}$. At this point, the full order state vector $\mathbf{X}$ of the original system can be approximately expressed as:

$$
\mathbf{X} \approx \Phi_{l} \mathbf{Z}
$$


The equation (27) is removed the subscript and substituted equation (12), we have

$$
\Phi \mathbf{z}_{k}=\mathbf{f}_{k}\left(\mathbf{u}_{k}, \Phi \mathbf{z}_{k}, \Phi \mathbf{z}_{k-1}\right)
$$

Equality both ends left multiply at the same time $\boldsymbol{\Phi}^{T}$

$$
\mathbf{z}_{k}=\boldsymbol{\Phi}^{T} \mathbf{f}_{k}\left(\mathbf{u}_{k}, \boldsymbol{\Phi} \mathbf{z}_{k} \boldsymbol{\Phi} \mathbf{z}_{-1 k}\right.
$$

This achieves the purpose of reducing the order of the original system, the dimension of the state variable is reduced from $n=2 N$ to $l=l_{p}+l_{s}$. The Newton iteration method is used to solve the nonlinear equations (29). Because of the low dimension of the state variables, the computational complexity can be greatly reduced.

\section{Example Verification}

A numerical example in the literature [11] is used. In this example, a two-dimensional oil-water two phase anisotropic reservoir is described. Its grid is divided into $21 * 21$, and the distribution of permeability and porosity is shown in Figure 1, 2. The related parameters of reservoir model: thickness $\mathrm{h}=2 \mathrm{~m}$, length and width of grid $\Delta x=\Delta y=33.33 \mathrm{~m}$, the viscosity of the crude oil $\mu_{o}=5 \mathrm{mPa} \cdot \mathrm{s}$, formation water viscosity $\mu_{w}=1 \mathrm{mPa} \cdot \mathrm{s}$, comprehensive compression coefficient $c_{t}=3.0 \times 10^{-3} \mathrm{MPa}^{-1}$, the original formation pressure $p_{i}=30 \mathrm{MPa}$, borehole radius $r_{\text {well }}=0.114 \mathrm{~m}$, the end point relative permeability of oil phase $k_{r o}^{0}=0.9$, the end point relative permeability of water phase $k_{r w}^{0}=0.6$, oil phase Corey index $n_{o}=2.0$, water phase Corey index $n_{w}=2.0$, residual oil saturation $S_{o r}=0.2$, irreducible water saturation $S_{w c}=0.2$. We use anti five point method well pattern to produce. Center has a water injection well, and four corners have four production wells. We ignore gravity and capillary force.

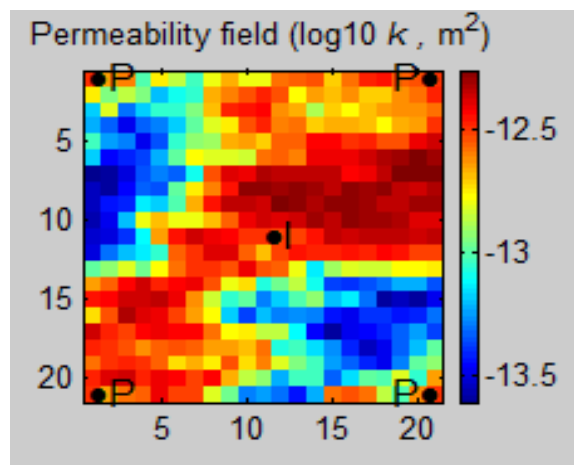

Fig.1 Permeability distribution of reservoir model

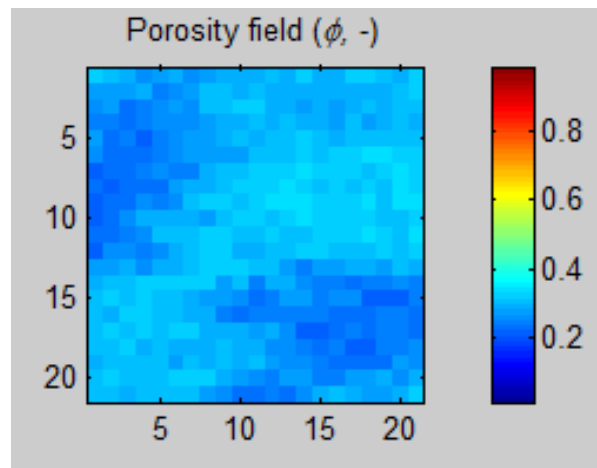

Fig.2 Porosity distribution of reservoir model

The numerical example is simulated by a fully implicit processing. We modify the source code to achieve POD model reduction process and verify the validity of the method. It is divided into training and forecasting two processes:

\section{(1) Training process}

The bottom hole pressure of production well is $29.5 \mathrm{MPa}$, the bottom hole flow of injection well is $0.0015 \mathrm{~m}^{3} / \mathrm{s}$. We run the full order simulator for 1400 days and save the results of the 66 time steps. The pressure matrix retains 37 singular values, saturation matrix retains 38 singular values, and the dimension of the base matrix $\boldsymbol{\Phi}_{l}$ of POD is $2 N \times l$, of which $l=37+38=75$. This means that the full order simulator is required to solve $2 N=882$ unknown variable, while the reduced order only needs to solve 75 variables.

In the training process, the comparison between the full order reservoir simulator and the reduced order simulator using POD method is shown in figure 3,4 . 


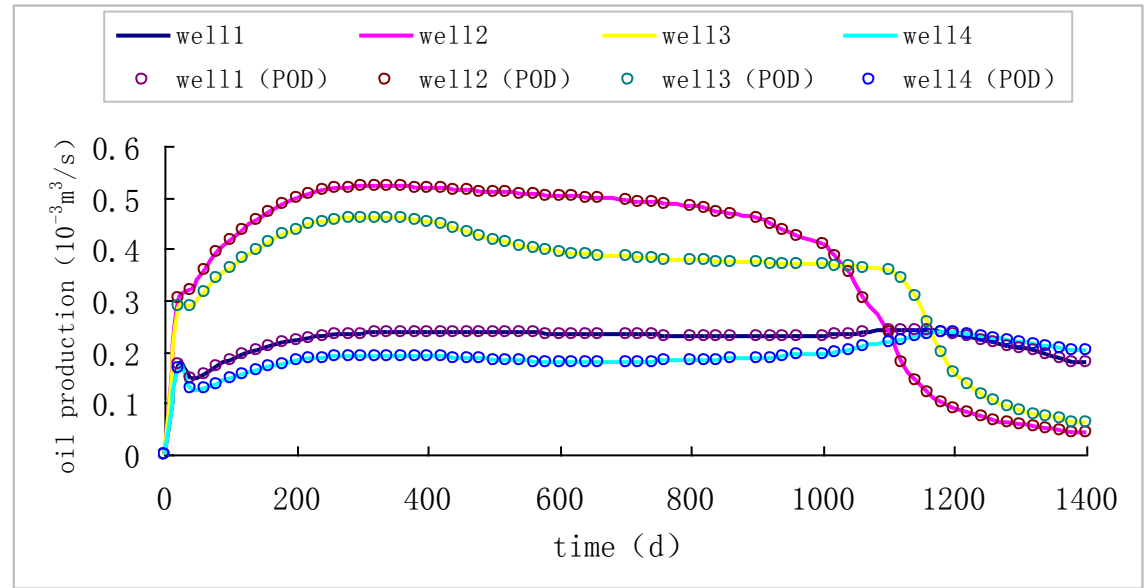

Fig.3 Oil production contrast of four production wells (training process)

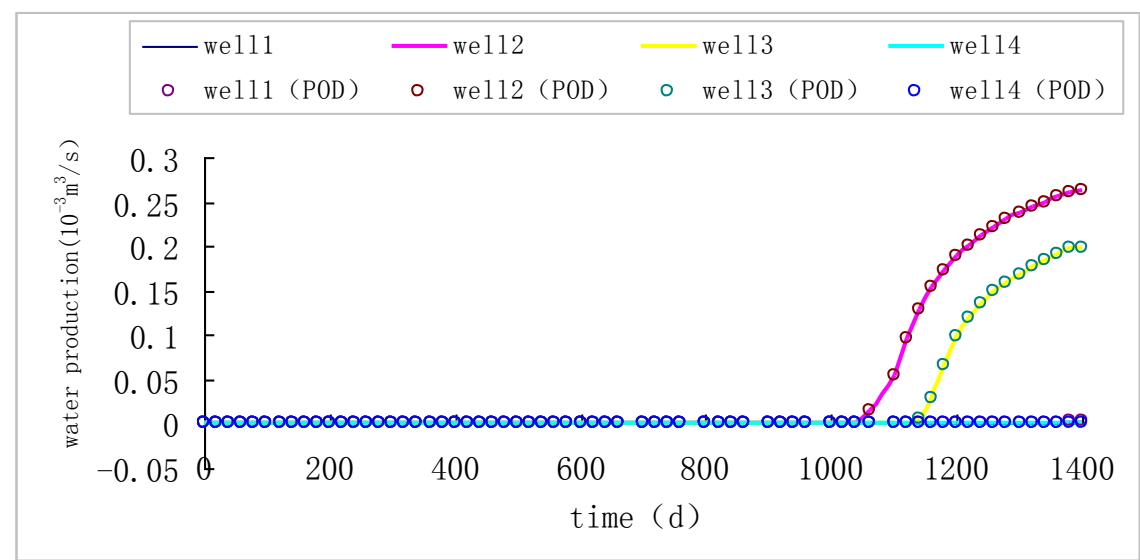

Fig.4 Water production contrast of four production wells (training process)

In this paper, the average relative error is used to measure the accuracy of the approximation. For example, the average relative error of oil production of per well is defined as:

$$
\bar{E}_{o}^{m}=\frac{1}{n_{t}} \sum_{i=1}^{n_{t}}\left|\left(\mathrm{q}_{o}^{m, i}-\mathrm{q}_{o, P O D}^{m, i}\right) / \mathrm{q}_{o}^{m, i}\right|
$$

Where $i$ indicates time step; $n_{t}$ indicates total number of time steps; $\mathrm{q}_{o}^{m, i}$ indicates the oil production of the full order simulator for the first $i$ step of production well $m ; \mathrm{q}_{o, P O D}^{m, i}$ indicates the oil production of the reduce order simulator for the first $i$ step of production well $m$. Similarly, the average relative error $\bar{E}_{w}^{m}$ of water production in each production well can be defined.

During the training process, the average relative error of oil production and water yield of four production wells is shown in Table 1.

Table 1 Oil production, water production average relative error of four production wells (training process)

\begin{tabular}{|ll|l|l|l|l|}
\hline \multicolumn{2}{|c|}{ Average relative error \% } & well1 & well2 & well3 & well4 \\
\hline $\bar{E}_{o}^{m}$ & 0.00726 & 0.01071 & 0.01032 & 0.01125 & \\
$\bar{E}_{w}^{m}$ & 0.01819 & 0.02315 & 0.0184 & 0.00001 & \\
\hline
\end{tabular}

The above results indicate that in the training process, oil production and water production of four production wells of reduce order and full order simulator are almost identical, the average relative error is very small, but the simulation time is increased nearly 3 times, the running time of the full order simulator is $35.527 \mathrm{~s}$, and the running time of reduction simulator is $12.019 \mathrm{~s}$. 


\section{(2) Forecasting process}

The base matrix $\boldsymbol{\Phi}_{l}$ obtained by the training process is used to verify the predictive ability of the POD reduced order model. At this time, the bottom hole pressure of production wells is changed to $28.5 \mathrm{MPa}$, and the flow rate at the bottom of the injection well remains unchanged. The comparison between the full order simulator and the reduced order simulator is shown in figure 5, 6 .

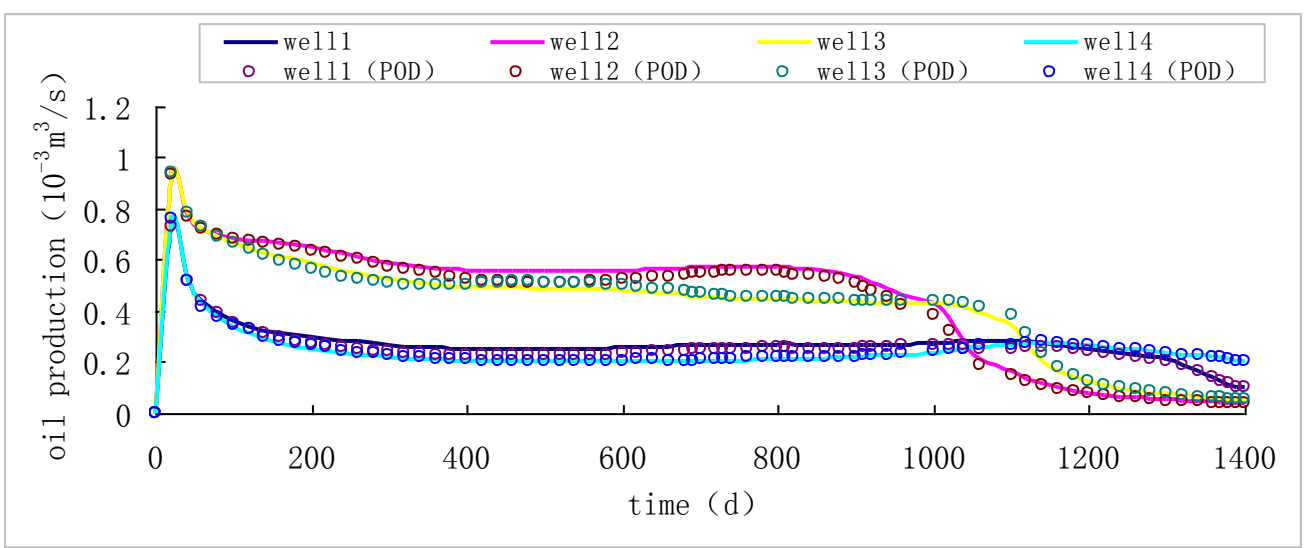

Fig. 5 Oil production contrast of four production wells (prediction process)

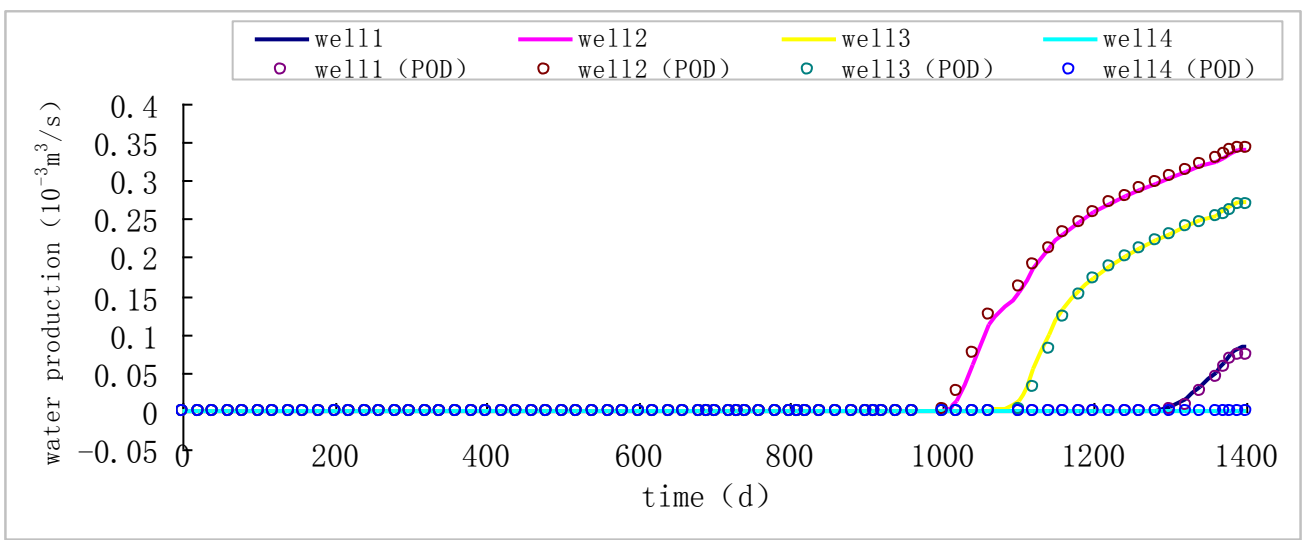

Fig. 6 Water production contrast of four production wells (prediction process)

In the forecasting process, the average relative error of oil production and water yield in four production wells is shown in Table 2 .

Table 2 Oil production、 water production average relative error of four production wells (prediction process)

\begin{tabular}{|lc|c|c|c|c|}
\hline \multicolumn{2}{|c|}{ average relative error \% } & well1 & well2 & well3 & well4 \\
\hline $\bar{E}_{o}^{m}$ & 4.8514 & 4.5452 & 2.9597 & 1.752 & \\
$\bar{E}_{w}^{m}$ & 2.5149 & 1.8732 & 2.0556 & 0.01 & \\
\hline
\end{tabular}

The results show that when the production schedule of forecasting process and training process are different, the average relative error of the reduced order and the full order simulator is improved, but it is still within the reasonable range of 5\%. At this time, the simulation time also increases by nearly 3 times. The full order simulator runs for $35.851 \mathrm{~s}$, and the running time of the reduced order simulator is $12.404 \mathrm{~s}$.

\section{Conclusion}

1) The application of POD model reduced order method to reservoir simulator can greatly reduce the dimension of reservoir model, and improve the operation speed of the simulator by nearly 3 times.

2) When the production schedule of the training and forecasting process is different, the average relative error of the reduced order simulator is improved, but still in a reasonable range of $5 \%$.

3) The improvement of the operation speed of the reservoir simulator provides an important solution for the practical application of the reservoir production optimization and history matching. 


\section{References}

[1]. J. D. Jansen, D. R. Brouwer, G. Navdal, et al. Closed-loop reservoir management [J]. First Break, $2005 ; 23$ (1):43-48.

[2]. Brouwer D, Jansen J. Dynamic optimization of waterflooding with smart wells using optimal control theory [J].SPE J, 2004, 9(4):391-402.

[3]. Chen Y, Oliver D, Zhang D. Efficient ensemble-based closed-loop production optimization [J].SPE J, 2009, 14(4):634-645.

[4]. Zhao Hui, Li Yang, Yao Jun, et al. Theoretical research on reservoir closed-loop production management [J].Sci China Tech Sci, 2011, 54(10):2815-2824.

[5]. P. Sarma. Efficient Closed-Loop Optimal Control of Petroleum Reservoirs under Uncertainty. PhD thesis, Stanford University, 2006.

[6]. T. Bui-Thanh, M. Damodaran, K. Willcox. Aerodynamic Data Reconstruction and Inverse Design Using Proper Orthogonal Decomposition [J]. AIAA Journal, 2004; 2(8):1505-1516.

[7]. M. Meyer,H. G. Matthies. Efficient Model Reduction in Non-linear Dynamics Using the Karhunen- Loeve Expansion and Dual-weighted-residual Methods [J]. Computational Mechanics, 2003; 31:179-191.

[8]. Y. Cao, J. Zhu, Z. Luo, et al. Reduced Order Modeling of the Upper Tropical Pacific Ocean Model Using Proper Orthogonal Decomposition [J]. Computers \& Mathematics with Applications, 2006; 52(8-9):1373-1386.

[9]. Roychowdhury J. Reduced-order Modeling of Time-varying Systems [J].IEEE Transactions on Circuits and Systems, 1999; 46(10):1273-1288

[10]. Khalid Aziz, Antonin Settari. Petroleum Reservoir Simulation [M]. London: Applied Science Publishers, 1979:128-133.

[11]. J. D. Jansen. Systems Description of Flow Through Porous Media [M].Springer: Springer Briefs in Earth Sciences, 2013:21-36.

[12]. Kerschen G, Jean-Claude Golinval, Vakakis A F, et a1.The Method of Proper Orthogonal Decomposition for Dynamical Characterization and Order Reduction of Mechanical Systems: an overview[J].Nonlinear Dynamics,2005;41:147-169.

[13]. Liang Y C, Lee H P, Lim S P, et a1.Proper Orthogonal Decomposition and Its App Cations-Part I: theory [J].Journal of sound and Vibration.2002;250(3):527-544.

[14]. Jiang Yaolin. Model Order Reduction Method [M].Beijing: Science Press, 2010:200-210. 